\title{
2D modeling of a Cepheid, moving grid approach
}

\author{
E. Mundprecht \\ Fakultät für Mathematik, Universität Wien, Nordbergstrasse 15, 1090 Vienna, Austria
}

\begin{abstract}
We present the first 2D simulation of a Cepheid of an effective temperature of 5125K.

The hydrodynamic equations in polar coordinates and their implementation in the ANTARES code
\end{abstract}

The equations governing the hydrodynamics are the continuity equation, the momentum density equation and the "total" energy density equation. Due to the size of the computational domain they are evolved in polar coordinates.

Continuity:

$$
\begin{aligned}
& \partial_{t} \rho=-\nabla \cdot(\vec{l}-\rho \vec{u}) \\
& \partial_{t} E=-\left(\nabla \cdot\left(\frac{\vec{l}}{\rho}\left(E+p-\sigma^{\prime}\right)-E \vec{u}\right)+\vec{l} \cdot \vec{g}+Q\right)
\end{aligned}
$$

Energy:

Momentum in radial direction: $\quad \partial_{t} I_{r}=$

$$
-\left(\partial_{r}\left(\frac{I_{r}^{2}}{\rho}-u I_{r}+p-\sigma_{11}^{\prime}\right)+\frac{1}{r} \partial_{\theta}\left(\frac{I_{r} I_{\theta}}{\rho}-\sigma_{21}^{\prime}\right)-\frac{2 I_{r}^{2}}{\rho r}+\frac{2 I_{r} u}{\rho}+\frac{I_{\theta}^{2}}{\rho r}+\frac{I_{r} I_{\theta}}{r \rho} \cot \theta+\sigma_{c o r r_{r}}+\rho g\right)
$$

Momentum in angular direction: $\partial_{t} l_{\theta}=$

$$
-\left(\partial_{r}\left(\frac{I_{r} I_{\theta}}{\rho}-u I_{\theta}-\sigma_{12}^{\prime}\right)+\frac{1}{r} \partial_{\theta}\left(\frac{I_{\theta}^{2}}{\rho}+p-\sigma_{22}^{\prime}\right)-3 \frac{I_{r} I_{\theta}}{r \rho}+\frac{2 I_{\theta} u}{\rho}+\frac{I_{\theta}^{2}}{r \rho} \cot \theta+\sigma_{\text {corr }_{\theta}}\right)
$$

with the momentum vector $\vec{l}=\left(I_{r}, I_{\theta}\right)$. In every vector, the first component denotes the radial direction, and the second the angular direction. The other variables are: the grid velocity $\vec{u}=(u, 0)$, the gas pressure $p$, and $\vec{g}=(g, 0)$ gravity. The radiative heating rate $Q$ is determined by the short-characteristic method (in the outermost region) and diffusion approximation, respectively. $\sigma^{\prime}$ is the viscous tensor in polar form.

All fluxes are computed at the cell centre and interpolated to the cell boundaries. For the hyperbolic part an ENO scheme with Marquina flux splitting (Fedkiw et al. 1996) is used. During the updating process the difference in cell volume due to the moving grid is included in the divergences and derivatives of the above equations. At the end of each time step the grid is advanced. The grid velocity $u^{\text {top }}$ at the top is determined as the horizontal average of the fluid velocity. The outermost grid point moves with $u^{\text {top }}$, the innermost grid point remains fixed, at the intermediate points the grid velocity and the grid points are computed via a dilatation factor. For details on the ANTARES code see Muthsam et al. (2007).

Up to now two models have been investigated, one with $T_{\text {eff }}=5125 \mathrm{~K}$ and one with $T_{\text {eff }}=5500 \mathrm{~K}$. Here, I present the cooler Cepheid. Some results including a bolometric light curve of the other star are presented by $\mathrm{H}$. Muthsam in the proceedings of symposium 7 of this JENAM conference. 

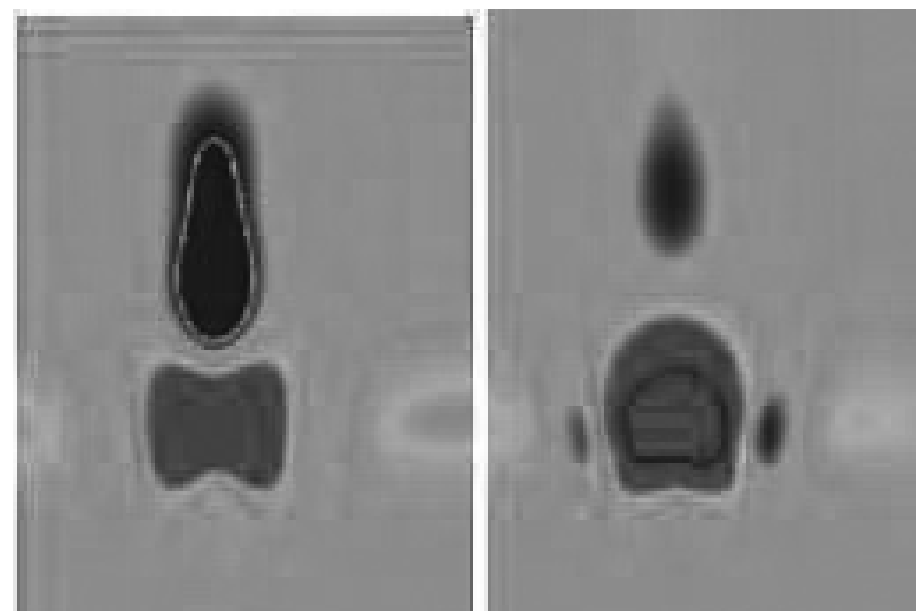

Figure 1: Convective fluxes $F_{c}$ : upwards red (black contours), downwards blue (white contours). Only one of nine convective cells at the very top of the computational domain is depicted. Left: contracted, right: expanded.

\section{Physical properties of the star and the domain}

Main Properties: $M=5 \mathrm{M}_{\odot}, L=912.797 \mathrm{~L} \odot, \log g=1.96, R=6.75 \mathrm{Gm}, P=4.16 \mathrm{~d}$, $(X, Y, Z)=(0.737,0.243,0.02)$.

The computational domain reaches from $T=4600 \mathrm{~K}$ to $T=320000 \mathrm{~K}$ and covers the top $42 \%$ of the star, with an aperture angle of $30^{\circ}$ and 450 horizontal grid points. An evenly stretched grid of 380 vertical grid points is a applied on the radial component, the cell height varies from $1.98 \mathrm{Mm}$ at the top to $125.3 \mathrm{Mm}$ at the bottom of the domain.

The simulation was started in 1D, after approx. 10 days regular pulsations were established. After 56 days a randomly perturbed angular momentum was added. The 2D simulation is now 34 days old. While the perturbations in the convective flux $F_{c}=I_{r}^{\prime} \rho^{\prime-1}(\rho h)^{\prime}$ still grow over time, correlations between the convective pattern and the phase of the pulsation are clearly visible. During the expansion the upwards flow increases and reaches a maximum shortly after the maximum radius, the downwards flow decreases. During the contraction the downwards flow increases, the upwards flow decreases. At the maximum expansion the temperature contrast between up- and down-flowing material is $\sim 890 \mathrm{~K}$ and thus smaller as at total contraction $(\sim 1400 \mathrm{~K})$. Also, the topology varies.

Acknowledgments. I am indebted to the Austrian Science Foundation for grants P18224 and P20762. G. Houdek, Univ. of Cambridge, kindly provided the hydrostatic starting model of the Cepheids and linear stability data.

\section{References}

Fedkiw, R. P., Merriman, B., Donat, R., \& Osher, S. 1996, Proceedings of the symposium on progress in numerical solutions of partial differential equations, Arcachon, France

Muthsam, H. J., Löw-Baselli, B., Obertscheider, C., et al. 2007, MNRAS, 380, 1335 